\title{
ANÁLISIS DE LA UTILIZACIÓN Y PRÁCTICA DE LOS MICROMACHISMOS POR LAS MUJERES EN MÉXICO
}

\author{
Carolina Hernández Jiménez ${ }^{1}$ \\ Dra. Josefina De la Cruz Izquierdo ${ }^{2}$
}

Resumen: En el presente artículo se realiza un análisis de una más de las violencias manifestadas en la actualidad contra la mujer por razón de género; la cual se diferencia de las demás por el tipo de práctica con la que se ejerce. Esta práctica es tan normal y natural que las personas no pueden notarla. Tiene una característica muy importante en cuanto al perfil del agresor, en la mayoría de las veces es ejercida por las mismas mujeres al realizar un comentario contra alguien más de su mismo sexo. Es importante enfatizar que dicha violencia inicia entre las mismas mujeres, principalmente aprendida desde el hogar, desde el momento en que, por ejemplo, las madres realizan la repartición de las actividades hogareñas correspondientes a los hijos e hijas. Se realizó un análisis concientizado de los micromachismos que existen y se concluyó que es una práctica tan común que incluso cuando las mujeres la vivencian o sufren alguna situación de este tipo no la perciben y no le dan importancia. Se analizaron algunos instrumentos y ordenamientos jurídicos establecidos para proteger a las mujeres de una vida libre y sin violencia, nos sólo a nivel nacional, sino también a nivel internacional.

Palabras Claves: Micromachismo, Violencia de Género, Igualdad, discriminación de Género.

\begin{abstract}
In the present article, an analysis of another representation of violence against women because of gender is realized; what makes the difference from the others is the type of practice which is exercised. This practice is so normal and natural that people cannot
\end{abstract}

\footnotetext{
${ }^{1}$ Estudiante del séptimo semestre de la Licenciatura en Derecho de la Universidad Juárez Autónoma de Tabasco.

${ }^{2}$ Doctora en Educación. Profesora Investigadora de la Universidad Juárez Autónoma de Tabasco.
} 
notice it. It has a very important characteristic regarding the profile of the aggressor, in most of the times it is exercised among women when making a comment against someone else of the same sex. It is important to emphasize that such violence begins among the same women, mainly learned from home, from the moment in which, for example, the mothers do the distribution of the corresponding housework for children. A conscious analysis of the micromachisms that existed was carried out and it was concluded that it is a practice so common that even when women experience it or suffer some situation of this type, they do not perceive it and do not give importance to it. Some instruments and legal systems established to protect women from a free and non-violent life were analyzed, not only at the national level, but also at the international level.

Keywords: Micromachism, Gender Violence, Equality, Gender Discrimination.

\section{Introducción}

El objetivo principal del presente estudio, es el análisis de los micromachismos existentes y analizar por qué son utilizados en su gran mayoría por las mismas mujeres, iniciando desde la misma ideología o estereotipo que se les ha enseñado desde el hogar, haciéndoles creer que los roles que les corresponden a ellas y ellos en la sociedad de ninguna manera es el mismo; por lo que, en la medida que se desarrollan para ellas se va volviendo una situación normal el recibir discriminación por razón de género; y más delicado aun que, ellas mismas se las aplican y las difunden, pues la visualizan como algo normal porque eso fue lo que se les enseñó y para lo que fueron educadas y formadas en sus hogares. En este sentido, la relevancia del análisis radica en que si bien existen estudios realizados que denominan al micromachismo como un tipo de violencia blanda y sutil en contra de la mujer que incluso fácilmente puede pasar desapercibido, no existen datos que como tal puedan dar perfil específico del agresor, pero sin duda alguna muchos autores coinciden en que si son ejercidos tanto por mujeres y hombres. Por tal razón se realizó una comparación de definiciones 
que coincidieron en algunas cuestiones del tipo de violencia; así mismo se realiza un estudio para definir quienes son las victimas de dicha violencia $y$ en que situaciones se puede presentar. Seguidamente se analiza quien o quienes son los agresores, es decir quien la práctica y la ejerce, buscando de igual forma en dicho apartado dar respuestas a tres interrogantes para definir: iel micromachismo es propio solamente de los hombres? ¿Todos los hombres lo practican? ¿Se origina desde el hombre? Posteriormente se realiza un análisis de los ordenamientos jurídicos protectores de las mujeres, aunque no existe como tal el termino en las leyes, lo cual no quiera decir que sea apropiado su uso. Cuando se expresa violencia en contra de la mujer, se engloban todos aquellos actos u omisiones basadas en la discriminación de la misma.

\section{Concepto De Micromachismo}

Algunos autores han definido los micromachismos coincidiendo en que es un tipo de violencia en contra de la mujer, siendo esta distinta a las que se escuchan comúnmente, ya que incluso en la mayoría de las veces no se perciben por la misma estrategia con la que se ejerce; tales autores lo definen como:

Las sutiles e imperceptibles maniobras y estrategias de ejercicio del poder de dominio masculino en lo cotidiano, que atentan en diversos grados contra la autonomía femenina. Hábiles artes, trucos, tretas y manipulaciones con los que los varones intentan imponer a las mujeres sus propias razones, deseos e intereses en la vida cotidiana. (Bonino, 2004)

Los micromachismos son actitudes de dominación "suave" o de "bajísima intensidad" formas y modos larvados y negados de abuso e imposición en la vida cotidiana. Son, específicamente, hábiles artes de dominio, comportamientos sutiles o insidiosos, reiterativos y casi invisibles que los varones ejecutan permanentemente. Son de tipo "micro" -tomando un término de Foucault-, del orden de lo capilar, lo casi imperceptible, lo que está en los límites de la evidencia. (Bonino, 2004)

Pequeñas tiranías cuasiimperceptibles, debido al grado de consentimiento de las mismas, que buscan demostrar la superioridad del 
hombre frente a la mujer", y a la que todavía hoy día se sigue considerando como el sexo débil. Se considera un tipo de "violencia blanda" que en el entorno de pareja puede ser el caldo de cultivo de relaciones vejatorias. (Vivero, 2017)

Son prácticas de dominación y violencia masculina en la vida cotidiana, comprenden un amplio abanico de maniobras interpersonales que impregnan los comportamientos masculinos en lo cotidiano. Son esos pequeños y cotidianos controles, imposiciones y abusos de poder de los varones en las relaciones de pareja, al que diversos autores (Miller, Bourdieu, Glick, Castañeda, etc.) han llamado pequeñas tiranías, terrorismo íntimo, violencia "blanda", "suave" o de "muy baja intensidad", tretas de la dominación, machismo invisible o sexismo benévolo. (Miravalles, S.F)

Por lo anterior se puede definir al micromachismo como un tipo de violencia de género, el cual consiste en que una persona ejerza dominio sobre otra mediante acciones, gestos, actitudes que muchas veces parecen tan normales que pasan desapercibidos, ya que se han naturalizado por lo frecuente que se utilizan en la vida cotidiana. Es una violencia que en muchas ocasiones se ejerce sin siquiera saberlo, ya que como mencionan los autores citados es de tipo blanda e incluso casi imperceptible, lo que quiere decir que apenas y puede ser percibido por la estrategia con la que se utiliza, pero que tiende a repercutir en la vida de la mujer, ya que estos, a pesar de no ser una violencia física, las victimas se va creando ideas y estereotipos en ella que permiten llegar a un control sobre ellas, dando pauta a la desigualad.

Este tipo de violencia se puede encontrar en todos lados y en todo tiempo debido a su naturaleza.

\section{Víctimas Del Micromachismo}

Este tipo de violencia de género es una forma de disfrazar el machismo, la cual como mencionaba, se puede encontrar todo el tiempo en la vida cotidiana, empezando desde el hogar, el trabajo, la escuela, en la familia, con los amigos, para ser más específicos en todos lados; misma que busca como tal demostrar el dominio del hombre hacia la mujer; es decir 
aquellos pensamientos, comentarios, actitudes, etc. Los cuales se ejercen de manera sutil para con la mujer, actualmente son normales y naturales que no se denota los efectos que pueden tener en las victimas de dicha violencia de género, es decir en "la mujer".

Su objetivo es anular a la mujer como sujeto, forzándola a una mayor disponibilidad e imponiéndole una identidad "al servicio del varón", con modos que se alejan mucho de la violencia tradicional, pero que tienen a la larga sus mismos objetivos y efectos: perpetuar la distribución injusta para las mujeres de los derechos y oportunidades. (Miravalles, S.F)

Con lo dicho queda claro que las mujeres son el blanco del micromachismo. Solo ellas son las víctimas de esta violencia. Lo que busca el micromachismo, como tal es seguir dominando a las mujeres pero utilizando nuevas técnicas y estrategias para ejercer dicho dominio de modo sutil que no pueda ser percibido; manipular por decirlo de alguna manera a la mujer haciendo que crean que son débiles y que el hombre es superior a ellas, por lo que deben depender de lo que digan o hagan; lo cual da pauta al trato desigual entre ellos y la aceptación por parte de ellas al mismo, este pensamiento es incluso, en muchas ocasiones, fomentado por los familiares del género femenino que no está consciente de esta violencia.

\section{Agresores Del Micromachismo}

El micromachismo, decíamos, es un tipo de violencia de género, con características específicas las cuales van enfocadas hacia la mujer y que están legitimadas por la sociedad. De igual forma como se ha comentado es una manera de demostrar la superioridad del hombre frente a la mujer y es esto lo que lleva a las interrogantes siguientes ¿el micromachismo es propio solamente de los hombres? ¿Todos los hombres lo practican?

La respuesta a dichas interrogantes es "NO", el micromachismo no solamente es propio de los hombres, así como toda violencia de género y no siendo 
esta la excepción puede surgir también por las mismas mujeres, porque desean agradar a los miembros masculinos de su entorno, que la han enseñado a mantener el dominio y superioridad del hombre y que cualquier "rebeldía" de las mujeres, debe reprimirse y retornar a los patrones ya establecidos para lograr mantener el "control” y orden (Ferrer Pérez, V., \& Bosch Fiol, E., \& Navarro Guzmán, C., \& Ramis Palmer, M., \& García Buades, M., 2008). La violencia es algo tan normal y tan naturalizado en la actualidad, que las mujeres adoptan criterios o alguna actitud hacia otras, sin siquiera saber que lo que está haciendo es promover el micromachismo, ejerciendo esta violencia de genero para con ellas mismas cuando no debería de ser así. Cuando se vive en una sociedad en la que muchas mujeres luchan día a día para el reconocimiento de sus derechos e igualdad en oportunidades en todos los ámbitos de la vida, y se encuentran en que no sólo se enfrentan a las ideologías de muchos hombres que están en contra de lo que ellas defienden, sino que, también tienen en contra las personas de su mismo género, crea una brecha de desunión entre las mismas, que incluso abre la pauta a una competencia insana. Es por eso por lo que las mujeres presentan un obstáculo incluso mayor al que presentan los hombres, que la lucha que muchas emprenden se representa de manera más complicada y dificulta el reconocimiento de la igualdad entre ambos géneros.

Tal como expresa (Yarzabal, s.f): "El micromachismo, al igual que toda forma de violencia de género, no es algo propio solamente de los hombres ni de todos los hombres. Son actitudes y pensamientos tan naturalizados en la sociedad que a veces surgen, incluso, desde las propias mujeres".

Es importante también aclarar que no todos los hombres practican este tipo de violencia sutil, de tal manera que no se puede generalizar; por ejemplo, en el caso de las mujeres, de igual forma no se puede afirmar que todas fomentan los micromachismos, pero el hecho que no sean todos no quiere decir que no exista; la cultura y la educación no es igual en todos los hogares, por lo que no todos aplican los diferentes tipos de violencia existentes. 


\section{¿Se Origina Desde El Hombre?}

Si bien es cierto, que de alguna manera repercute el poder que se le ha dado a los hombres en la familia desde hace muchos años atrás, desde que se habla del patriarcado donde el hombre era el jefe de la familia y quien tenía el derecho sobre todos los integrantes de la misma, esta culpa no solo le corresponde a él, porque la mujer también ha sido participe en el origen de este tipo de violencia de género, por imitación o ignorancia y desgraciadamente en algunas ocasiones, se ha instaurado como la actora principal. Como se había mencionado anteriormente, esto es un problema que nace desde la educación que se recibe en la casa. Desde el hogar, cuando son muy pequeños se les dan roles distintos a los niños y a las niñas, y desde ahí inician las enseñanzas de baja valía y sometimiento que se inculcan y que tienen un efecto muy negativo en la formación de los hijos. En la medida en la que crecen esa desigual división de los roles continua y se enfatiza. A la joven se le asignan las labores del hogar en general y se le enseña a manejar el control de la casa, a no tener voz ni voto, ni poder de decisión, a ceder las manipulaciones en general por el "bien" de los hijos, a sufrir críticas y desvalorizaciones y ser castigada o ignorada si el comportamiento no era el "aceptado"; en cuanto a los jóvenes, se les enseña a ocuparse de arreglos mecánicos y manuales de construcción y reparación de la casa, al igual que a realizar trabajos donde se utilice la fuerza, a tomar todas las decisiones referentes al control y manejo de la casa y sus integrantes, a controlar el dinero y por ende, decidir, a recibir atenciones y demandar su "derecho" a recibirlas, imponer sus gustos, su tiempo y sus cambios de humor, etc. Dichas conductas han sido enraizadas en sus subconscientes, por lo que, si ven que alguien sale del parámetro de lo establecido, es rechazada y violentada para que se "comporte a cómo debe hacerlo". Es por ello por lo que cuando las mujeres se encuentran ante una situación sutil, como lo son los micromachismos, no la notan, ya que les parece algo normal y natural, porque todas sus vidas les han vendido esas ideas como correctas, que ese el comportamiento apropiado, en donde el 
hombre es superior a ellas, por ser fuertes y proveedores, por lo tanto, su deber es procurarlo $\quad \mathrm{y}$ atenderlo. Desafortunadamente, entre las mismas mujeres, se estila que la madre enseñe a su hija y la prepare para que sea buena esposa y buena madre; la dispone para atender a su esposo y a los hijos, inculcándole que es ella quien debe realizar las labores del hogar por el simple hecho de ser mujer y por el bien de la familia. Es por esto por lo que, esta violencia se considera más que nada cultural y educacional. Muy aparte de los roles establecidos, también se les ha enseñado a las mujeres a luchar todas contra todas, a buscar ser mejor unas que las otras, a vivir en enemistad las unas con las otras, por el sólo hecho de ser individualistas, de modo que no se pueden unir para una misma causa. Cuando una logra escalar y le surge una oportunidad en la vida, las críticas y los comentarios discriminatorios vienen de ellas mismas, porque se le ha enseñado a que las mujeres no valen, y no deben ser reconocidos sus esfuerzos y lo que son capaces de lograr con sus propios méritos. Viven con la idea que se les ha hecho creer en donde, el hombre se supone superior a ellas y que, para lograr algo siempre los van a necesitar, empoderando de esta forma más al hombre. Esto permite y da pauta a la violencia de género que las mantiene sometidas.

\section{Micromachismos Más Comunes}

Conductas micro machistas se encuentran en todas partes, y que muchas veces no se perciben como tal, ya que, la sociedad esta tan acostumbrada a ciertos comentarios y acciones que no les da mayor importancia y lo deja pasar desapercibido. Sin embargo, incluso muchas veces las personas que lo ejercen no son conscientes de ello.

Algunos ejemplos muy simples son los siguientes:

1. Distinguir entre señorita y señora

2. Negarse rotundamente a que una mujer te ceda el paso

3. Asumir que los hombres solo merecen lo mejor o lo más fuerte

4. Recibir comentarios por parte de camareros que te dicen guapa y preciosa

5. Escuchar frases como: "Déjame que tú no sabes"; o: "quita que yo 
Periódico do Núcleo de Estudos e Pesquisas sobre Gênero e Direito Centro de Ciências Jurídicas - Universidade Federal da Paraíba V. 7 - $\mathrm{N}^{\mathrm{o}} 04$ - Ano 2018 - Spanish Edition ISSN | 2179-7137 | http://periodicos.ufpb.br/ojs2/index.php/ged/index

lo acabo más rápido" que contribuyen a demeritar el accionar de la mujer frente al hombre.

6. Dominar con el silencio $Y$ así decidir cuándo y cómo se hablará de algo. Suele ir acompañado de un "eres una histérica" o un “estás exagerando”, recalcando que la mujer es una sentimental e impulsiva incapaz de razonar.

7. Saludar a ella con beso, pero darle la mano a él

8. Los pañales son cosas de mujeres. Los hombres, aunque quieran no pueden porque ni siquiera hay un espacio donde puedan hacerlo.

9. Sentarse holgadamente en transportes públicos (Manspreading) con piernas abiertas y limitar el espacio a las mujeres.

10. "Vaya modelito" En las bodas, en las graduaciones, en las entregas de premios, etc. se comenta solo la indumentaria de las mujeres y se realizan críticas muy duras a quien se sale de la norma. Por supuesto, ellos irán cómodos y ellas vestidas y con tacones.

11. "Sonríe que estás muy seria"

12. Nombres distintos en las mismas profesiones

13. Las mujeres pagan menos en las discotecas. No es discriminación positiva, es tratar a las mujeres como ganado, ellas son el producto.

14. "Hoy te han dejado de niñera" No es una niñera, es un padre, cuida a su hija porque es su responsabilidad y no porque hoy su mujer estaba ocupada.

15. El comentar que una mujer no se ha depilado

16. Uniformes con falda para ellas y pantalones para ellos

17. "Tan fuerte como Superman" Cuando se alaban habilidades de niños y niñas se tiende a usar modelos masculinos ¿acaso no hay mujeres fuertes o rápidas?

18. Regalar cosas diferentes a niños y niñas

19. "Nenaza” Hacer algo como una mujer es un insulto. 
20. "Así no se comporta una señorita"

21. 25. “¿Y tú ya tienes novio?” es el “¿y tú para cuándo?” de las bodas. El hetero patriarcado en todo su esplendor: niñas que tienen que pensar en buscar a su príncipe azul (princesa no vale) y no muy tarde. ( Murnau, 2016)

Todos los ejemplos analizados son cuestiones que fácilmente se pueden encontrar todos los días y en cualquier espacio de la vida cotidiana, dígase la escuela, el hogar, el trabajo, en espacios públicos, en la familia y con amistades; comentarios que al escucharlos aparentemente no tienen ninguna mala intención o no causan ningún efecto en las personas a las cuales se les hace. Incluso se dicen sin pensarlo por el momento en el que se encuentran y parece que viene bien comentar algo, y sin pensarlo lo mencionan, ya sea porque lo hayan escuchado antes o es tan normal que inclusive lo habrán hecho en ocasiones anteriores y no pasó nada, siendo así más fácil volver a decir algo igual o parecido. Cuando se menciona "Manspreading" dicho término se refiere a la costumbre o practica de los hombres de sentarse con las piernas abiertas en el transporte público ocupando más espacio del normal. Es fácil de observar en cualquier transporte que las mujeres ocupan espacios reducidos, sentándose con las piernas cruzadas o juntas y los hombres que van a su lado llevan las piernas abiertas ocupando más un asiento; de tal manera que ellas apenas y pueden moverse. Con lo mencionado, es evidente la diferencia que hay entre la manera que se comportan una mujer y un hombre en los espacios públicos, la práctica del Manspreading, implica que él puede ocupar todo el espacio que quiera sin tomar en cuenta el espacio de ella; en cambio la mujer debe limitarse, considerando esa práctica como si ella tuviera que pedir permiso para poder sentarse. Con respecto a esta práctica, en las redes sociales se realizaron diversas campañas, una de ellas fue: \#tuspelotasnosontangrandes, llevada a cabo en Nueva York y Seattle. Se dio una gran controversia que cuestionaba por qué molesta tanto, ¿Tan difícil es que se sienten de manera normal y respetuosa? Esta situación trae implícito que no se trata si sobra o no espacio, el fondo es la actitud 
que ejerce dominación de poseer ese lugar por decreto, por el simple hecho de ser hombre; es decir, una declaración corporal sobre el espacio público, sobre aquel supuesto espacio que él cree que se merece por sobre de ella; como tal, éste no presenta el gran problema al que la mujeres se enfrentan, pero si es un punto más que anexar a la infinita lista de momentos cotidianos donde el varón ejerce su dominio sobre ella, haciendo notar su superioridad. (Marnau, 2015)

De igual forma, en el listado se menciona que los pañales son cosas de las mujeres. Esto es otra cuestión que hace notable la desigualdad que existe entre el hombre y la mujer y el rol que se le ha dado a ella como la cuidadora de los niños, ya que nunca se ha visto en los baños de los hombres el icono asignando un lugar para que se le cambie de pañales a los bebes. Esa actividad le corresponde exclusivamente a las mujeres, por ello en el baño de ellas se encuentran siempre el lugar asignado para cambios de pañal; este hecho tan normal y al cual estamos tan acostumbrado que pasa desapercibida la discriminación y el estereotipo que implica y una vez más se demuestra el dominio que se tiene hacia ellas, ya que se les determina que ellas son cuidadoras, por lo que deben atender a sus hijos y al esposo, pues no puede realizar ninguna otra actividad pues son las madres. Otro ejemplo de micromachismos es el regalar cosas distintas a las niñas y a los niños, lo cual ya se había mencionado, a las niñas se les regalan los juegos de té y de cocina, la casita, las muñecas, etc. Por el contrario, a los niños se les regalan un balón, muñecos de súper héroes, carros, herramientas, entre otras cosas. Con dichos regalos los niños y las niñas se van creando una identidad, ya que, a ellas, desde pequeñas se les enseña que son las encargadas del hogar y por ello deben atender a los hijos y a su esposo; cuando llega a cierta edad empieza a hacer realidad todo lo que jugaba, se encarga de aprender las labores del hogar, su madre se encarga de prepararla para que sea una buena esposa, etc. En cuanto al niño al darles dichos juguetes y dejarle salir a jugar a la calle se le enseña que él es capaz de salir a la calle a cualquier hora, tener el control en la casa y ser el encargado de traer el dinero a casa. Por lo regular, los padres no razonan al regalar estos juguetes a sus hijos. No saben 
que están manipulando su identidad desde que son pequeños y les están enmarcando en un estereotipo de género que con el paso del tiempo tendrá sus consecuencias. Es importante analizar como una cuestión que parece que no tiene ninguna importancia, que es algo tan normal y por lo mismo pasa desapercibido, crea un tipo de violencia sutil, blanda que incluso no se percibe, pero tiene como efecto empoderar cada vez más al hombre, haciéndole ver o creer su superioridad y dominar por sobre la mujer; ciertamente dichas acciones son estrategias de dominio del hombre y un ejemplo de la desigualdad que existe entre ambos géneros.

Otras frases micro machistas tan normalizadas, comunes y cotidianos, son cuando:

Te dicen que deberías ser un poco más femenina.

$\mathrm{Tu}$ pareja te dice que no te pongas esa ropa tan provocadora.

Te dicen que deberías conseguirte un novio que te haga feliz.
Te dicen debes cuidar tu figura porque nadie se fijaría en alguien como tú.

Nadie admite que quizá no quieres a más nadie que a ti misma para ser feliz.

Que aprendas a cocinar para tu marido, cuando te cases.

Te piden que hagas las labores del hogar, sólo porque eres la mujercita de la casa.

Te dicen que tú no deberías de opinar de fútbol, porque eso es cosa de hombres.

Te dicen que no tengas tanto carácter porque no es propio de una señorita.

Te miran mal por decir algo fuera de lugar para una señorita.

Te gritan por la calle y sabes que no precisamente piropos. Te dicen ilusa por pensar que un hombre como él se fijaría en alguien como tú.

De niña te vestían de princesita, aunque, tú te querías disfrazar de superhéroe. 
Te dicen perra porque no quieres una relación estable.

\section{Internacional}

(Yarzabal, s.f)

En materia internacional existen

\section{Leyes que sancionan la discriminación} y violencia contra la mujer.

\section{Culturalmente el micro} machismo esta implementado, insertado dentro de las tradiciones y educación de las familias, y aunque se considera violencia de género, la misma sociedad "obliga" a que se mantenga esta discriminación de la mujer, por lo que es imperante conocer los instrumentos jurídicos que puedan prevenirlo, sancionarlo o erradicarlo. En sí, no existe un ordenamiento jurídico en el que se estipulen sanciones específicamente a los micromachismos en sí y como tal, pero sí existen leyes, normas, convenciones y tratados internacionales que protegen a la mujer contra todo tipo de violencia que debe ser entendido como cualquier acto u omisión que es basada en la discriminación en contra de la misma, por razones de género. Por lo mismo realizaremos un análisis de tipo internacional y nacional revisando dichos instrumentos jurídicos. varios convenios y tratados que garantizan la protección de una vida libre y sin violencia de las mujeres, para que de esta manera ellas se encuentren salvaguardadas de todo tipo de violencia por género. Dichos instrumentos son reconocidos por la Constitución en su primer artículo en donde se puede interpretar que los tratados tendrán la misma jerarquía que la Constitución Política de los Estados Unidos Mexicanos (CPEUM) ven materia de derechos humanos. Aunque ciertamente esto no ha logrado impedir que se sigan suscitando este tipo de cuestiones tales como los son los micromachismos. Algunos tratados internacionales son:

- Declaración Universal de los Derechos Humanos

En esta Declaración en su primer artículo se establece que todos los humanos nacen libres e iguales en derechos, por lo que no debe existir 
diferencia de trato entre los hombres y las mujeres, ya que toda persona tiene los mismos derechos que son proclamados por dicha Declaración sin ningún tipo de diferencia, dígase, sexo, raza, religión entre otras cuestiones que muchas veces suelen ser causa de distinción. Este mismo ordenamiento estipula de igual forma que, todos tendrán, de la misma forma, el derecho a la protección contra la discriminación (Naciones Unidas, 2015)

Otra cuestión importante es lo que se establece en su artículo 23 que al texto dice: "Toda persona tiene derecho al trabajo, a la libre elección de su trabajo, a condiciones equitativas y satisfactorias de trabajo y a la protección contra el desempleo" (Naciones Unidas, 2015). Es así como con esta convención se busca la igualdad entre los hombres y las mujeres, por lo que lo establecido en el ordenamiento debe ser aplicado de manera igualitaria.

- Convención para la Eliminación de todas las Todas las Formas de Discriminación contra la Mujer o CETFDCM
Con esta convención lo que se pretende es lograr la plena igualdad entre el hombre y la mujer, ya que la discriminación para con las ultimas ha rebasado todos los límites y violado los principios de igualdad de derechos y el respeto a una vida digna; por ello, reconociendo todos los esfuerzos de ellas y el papel tan importante que tienen en la sociedad y en el núcleo familiar, así como los grandes e importantes aportes de las mismas; por ello, para modificar toda esa desigualdad, se ve la necesidad de cambiar el papel tradicional no solo de la mujer sino que también del hombre en la sociedad y en la familia. Algunos puntos muy importantes del mencionado ordenamiento son:

Artículo 5. Los Estados Partes tomarán todas las medidas apropiadas para:

a) Modificar los patrones socioculturales de conducta de hombres y mujeres, con miras a alcanzar la eliminación de los prejuicios y las prácticas consuetudinarias y de cualquier otra índole que estén basados en la idea de la inferioridad o 
superioridad de cualquiera de los sexos o en funciones estereotipadas de hombres y mujeres;

b) Garantizar que la educación familiar incluya una comprensión adecuada de la maternidad como función social y el reconocimiento de la responsabilidad común de hombres y mujeres en cuanto a la educación y al desarrollo de sus hijos... (Organización de las Naciones Unidas, 1981)

Los Estados que sean parte también deberán tomar las medidas necesarias para que no exista discriminación en la vida política de la mujer y; en caso de que haya, tendrá que eliminarla y garantizar la participación de la mujer en la vida política, existiendo igualdad de condiciones entre los hombres, en cuanto a sus derechos políticos dentro de ellos de votar y ser votada y participar en la creación de políticas públicas o en otros casos ocupar cargos públicos. En su artículo 9 establece que la nacionalidad del marido no tendrá porque modificar la de ella, así haya sido matrimonio con un extranjero. En lo que respecta al ámbito educacional y laboral, los Estado deberán garantizar que exista un trato igualitario tomando todas las medidas necesarias para que no se suscite ningún tipo de discriminación y, en caso de que la haya, será su obligación eliminarla. (Organización de las Naciones Unidas, 1981)

- Declaración Americana de los Derechos y Deberes del Hombre

Específicamente en su Artículo 2, relacionado con el Derecho de igualdad ante la Ley que dice: "Todas las personas son iguales ante la Ley y tienen los derechos y deberes consagrados en esta declaración sin distinción de raza, sexo, idioma, credo ni otra alguna" ( Conferencia Internacional Americana, 1948).

- La Convención Interamericana para Prevenir, Sancionar y erradicar la Violencia Contra la Mujer

a) En la presente Convención se entiende por violencia contra la 
mujer "cualquier acción o

conducta, basada en su género, que cause muerte, daño o sufrimiento físico, sexual o psicológico a la mujer, tanto en el ámbito público como en el privado" (Organización de los Estados Americanos, 1995).

Así también, se protegen los derechos de la mujer a una vida libre y sin violencia tanto en lo que concierne a lo Público y lo Privado, a la protección de todos los derechos que le dan cada uno de los ordenamientos, tanto regionales como internacionales; por lo que puede ejercer sus derechos, políticos, económicos, sociales y culturales que se encuentren en los instrumentos jurídicos. Y en el Artículo 6 establece el derecho de toda Mujer a una vida libre de violencia que incluye, entre otros:

b) El derecho de la mujer a ser libre de toda forma de discriminación, y

c) El derecho de la mujer a ser valorada y educada libre de patrones estereotipados de comportamiento y prácticas sociales y culturales basadas en conceptos de inferioridad o subordinación. (Organización de los Estados Americanos, 1995)

\section{Nacional}

- Constitución Política de los Estados Unidos Mexicanos

La constitución en el párrafo quinto de su artículo protege a la mujer contra la violencia al establecer:

Queda prohibida toda discriminación motivada por origen étnico o nacional, el género, la edad, las discapacidades, la condición social, las condiciones de salud, la religión, las opiniones, las preferencias sexuales, el estado civil o cualquier otra que atente contra la dignidad humana y tenga por objeto anular o menoscabar los derechos y libertades de las personas. (Cámara de Diputados del H. Congreso de la Unión, 1917)

En el artículo 2 de la misma ley se les otorga libre determinación y por ende 
autonomía a los pueblos indígenas, para que puedan decidir sus propio sistema normativo y regulación de sus conflictos internos, pero esta autonomía es limitada por la misma constitución al establecer que deberá sujetarse "a los principios generales de esta Constitución, respetando las garantías individuales, los derechos humanos y, de manera relevante, la dignidad e integridad de las mujeres". De igual forma, en el mismo artículo establece que en cuanto a la elección de sus autoridades o representantes para su gobierno interno, que deberán elegirse "garantizando que las mujeres y los hombres indígenas disfrutarán y ejercerán su derecho de votar y ser votados en condiciones de igualdad". Con las limitaciones mencionadas se evita dar pauta a prácticas discriminatorias, protegiendo así el desarrollo de la mujer en los pueblos mencionados. En el artículo 4 de la CPEUM mediante el principio de igualdad se garantiza de igual forma protección a las mujeres, lo que al texto dice "El varón y la mujer son iguales ante la ley". (Cámara de Diputados del H. Congreso de la Unión, 1917)

\section{Leyes Federales}

Dentro de las leyes federales existentes que buscan la protección de la mujer y la eliminación de toda discriminación por causa de género se hallan:

- Ley General de Acceso de las Mujeres a una Vida Libre de Violencia

- Ley General para la Igualdad entre Mujeres y Hombres

- Ley General de Educación

- Ley General de Salud

- Ley Federal del Trabajo

- Ley General de Instituciones y Procedimientos Electorales.

Con las leyes antes mencionadas, en México se busca la protección a la mujer para que pueda ser libre de todo tipo de violencia de género y discriminación, estableciendo en los ordenamientos la igualdad que debe de existir en la aplicación de las mismas, tanto a los hombres como a las mujeres. En el caso de Ley General de Educación, el Estado tiene la obligación de que la educación que impartan se imparta en base a la igualdad y la inclusión, cuidando de eliminar la 
creación de estereotipos y la violencia que se da en contra de la mujer, niñas y niños. En cuanto a las cuestiones laborares en la norma referente a ella, se garantiza la igualdad de condiciones entre ambos; así como también la igualdad en obligaciones y no hay que dejar de lado que actualmente la mujer puede participar en cuestiones políticas en las mismas condiciones del hombre ya que la ley garantiza la paridad de género.

\section{Resultados}

El micromachismo es un hecho ya identificado en la sociedad actualmente $\mathrm{y}$, por lo ya mencionado anteriormente, se puede conocer que este es un tipo más de violencia en contra de la mujer por razón de género, que fortalece los otros tipos de violencia más evidentes. Dicha violencia se origina por la gran desigualdad existente en la educación impartida a los hombres y las mujeres debido a los estereotipos y roles asignados, de manera diferente a cada uno. También se definió que estas actitudes micro machistas son asignadas incluso por las mismas mujeres. Estas actitudes e incluso enseñanzas traen incluida la desvalorización y baja autoestima en la mujer, agotamiento emocional, depresión y hartazgo; cuestiones que implican un control y manipulación sutil que buscan el conservar la posición de dominio o superioridad al hombre y afirmar su identidad masculina. Desgraciadamente este tipo de comportamientos se soslayan, se aceptan y se dejan pasar porque, debido a los estereotipos son algo aceptado por la sociedad y las mismas mujeres lo aplican entre ellas mismas de manera inconsciente, con el único propósito de "ayudar" a otras a seguir las pautas marcadas por la sociedad, aunque estas violenten su dignidad y desarrollo personal. Manejando como pretexto la realización de esto, el que se nos haya inculcado el tener siempre cuidado de nuestro aspecto y querer agradar a otros, el siempre estar en la búsqueda de esa persona que resolverá todos los problemas que enfrentemos, que debemos luchar por mantener una relación por salvar la "familia", haciendo hasta lo imposible por retener esa relación y sólo así estar completa. Es por todo ello que es posible concluir que el micromachismo es en gran 
parte originado y fomentado en la educación impartida desde chicos, así como llevado a la práctica también por las mujeres; ciertamente los hombres con sus acciones, tretas, comentarios y estrategias la ejercen y sus manifestaciones son muy obvias, pero esas actitudes y comportamientos no es algo propio solo de ellos, sino que también de ellas de manera automática, aún sin saberlo y sin darse cuenta, día a día la llevan a cabo, regida por los mismos estereotipos sociales que se les han inculcado, tanto que cuando una mujer sufre de micromachismos no lo mota porque es algo normal y natural para ella. Por lo que es importante que sea abordada de carácter urgente, que se ventile y se dé a conocer, ya que al ser tan sutil es muy fácil que se siga ocultando en la sociedad y en la vida cotidiana, y que poco a poco se haga más fuerte y siga causando daño, aun cuando existen diversos ordenamientos jurídicos protectores de la mujer para que tenga una vida digna, libre y sin violencia. Por ello es importante tomar las medidas necesarias para darlos a conocer, educar para lograr la igualdad y seguir luchando por que dichos ordenamientos se apliquen apropiadamente y logren dar los resultados que se requieren para la implementación de una sociedad incluyente e igualitaria.

\section{Conclusión}

Es importante definir ciertas pautas necesarias para erradicar los micromachismos, como, por ejemplo, el de darle difusión a los estudios e investigaciones realizados acerca de esta temática y no solo los estudios que son realizados dentro del mismo país, sino que también a nivel internacional, para que esta información se haga llegar a todos los sectores de la sociedad, así de esta forma deje de ser visto como algo normal y natural. Se debe buscar que el Estado realce y lleve a cabo políticas públicas mediante las cuales se concientice a la sociedad de la violencia que se ejerce de manera normal y cotidiana en contra de las mujeres en nuestro país, para que así se pueda combatir y eliminar no solo el micromachismo, sino que también toda violencia de género. En cuanto a cuestiones académicas, los modelos educativos deben revisarse para definir si no se están implementando 
micromachismos ocultos en las escuelas y garantizar que con la educación en las aulas se está combatiendo todo tipo de discriminación de género. Al ingresar en los hogares se busca de igual manera el reeducar, consistiendo esto en que cada persona inicie desde sus hogares con la concientización y la eliminación de todo tipo de discriminación de género, la cual se podrá lograr en primer lugar evitando hacer la repartición de los roles entre los hombres y las mujeres de manera distinta, así como educar a las hijas e hijos sin perspectiva de género, recalcando el respeto y la igualdad.

\section{Bibliografía}

Conferencia Internacional Americana. (2 de Mayo de 1948). Declaracion Americana de los Derechos y Deberes del Hombre. Recuperado de Declaracion Americana de los Derechos y Deberes del Hombre:

https://www.oas.org/dil/esp/Declaraci\%C 3\%B3n_Americana_de_los_Derechos_y_ Deberes_del_Hombre_1948.pdf
Murnau, M. (28 de Enero de 2016).

Feminista Ilustrada. Recuperado de 25

Micromachismos:

https://feministailustrada.com/2016/01/28

/25-micromachismos/

Bonino, L. (2004). Los Micromachismos.

La Cibeles $N^{\circ} 2$ del Ayuntamiento de

Madrid, 1. Recuperado de

http://www.mpdl.org/sites/default/files/mi

cromachismos.pdf

Cámara de Diputados del H. Congreso de la Unión. (5 de Febrero de 1917). Contitución de los Estados Unidos Mexicanos. México, México. Recuperado de

http://www.diputados.gob.mx/LeyesBibli

o/pdf/1_150917.pdf

Marnau, M. (9 de Noviembre de 2015).

Cuidado con cómo te sientas en el metro.

Recuperado de Feminista Ilustrada: https://feministailustrada.com/2015/11/09 /cuidado-con-como-te-sientas-en-elmetro/

Miravalles, J. (S.F). Gabinete de Psicología. Recuperado de Sexismo 
Amibivalente:

http://www.javiermiravalles.es/Sexismo\%

20Ambivalente/E1\%20Machismo\%20y\%

20los\%20Micromachismos.html

Naciones Unidas. (2015). Declaración

Universal de Derechos Humanos.

Recuperado de Declaración Universal de

Derechos

Humanos:

http://www.un.org/es/documents/udhr/U

DHR_booklet_SP_web.pdf

Organización de las Naciones Unidas. (12 de Mayo de 1981). Convención sobre la Eliminación de Todas las Formas de Discriminación contra la Mujer. Recuperado de Convención sobre la Eliminación de Todas las Formas de Discriminación contra la Mujer: http://cedoc.inmujeres.gob.mx/documento s_download/100039.pdf

Organización de los Estados Americanos. (14 de Agosto de 1995). La Convención Interamericana para Prevenir, Sancionar y erardicar la Violencia Contra la Mujer. Recuperado de La Convención Interamericana para Prevenir, Sancionar y erardicar la Violencia Contra la Mujer: https://www.oas.org/dil/esp/convencion_ belem_do_para.pdf

Vivero, V. (27 de Febrero de 2017).

Doctissimo. Recuperado de Micromachismos: qué son y cómo detectarlos:

http://www.doctissimo.com/mx/bienestar/ psicologia/relaciones-

sociales/micromachismos-que-son

Yarzabal, L. (s.f). VIX Inc. Recuperado de Micromachismo: 20 formas de violencia de género que suelen pasar desapercibidas en la vida cotidiana: https://www.vix.com/es/btg/curiosidades/ 60120/micromachismo-20-formas-deviolencia-de-genero-que-suelen-pasardesapercibidas-en-la-vida-cotidiana 